\title{
Microcavities in Semiconductor Materials
}

A. van Veen, R.A. Hakvoort, H. Schut and P.E. Mijnarends

Interfaculty Reactor Institute, Delft University of Technology, Mekelweg 15, NL-2629 JB Delft, The Netherlands

\begin{abstract}
Positron beam and helium desorption techniques have been applied to different materials, in particular semiconductor materials, to determine the presence of defects. The positron technique yields values of the positron diffusion length and values of the Doppler broadening parameters. In principle, defect concentrations can be derived and an indication can be obtained about the nature of the defect. Results are presented which show that cavities can be easily detected. It is also demonstrated that gas accumulated in the cavities reduces the observed differences between the defected and the defect-free material. Large cavities were detected in solar cell hydrogenated amorphous silicon and low temperature deposited amorphous silicon. It was found that layers deposited under irradiation with low energy ions (ion assisted deposition) did not show evidence of microcavities. Desorption techniques were successfully employed to detect cavities in silicon.
\end{abstract}

\section{INTRODUCTION}

Positrons have a high affinity for trapping in open volume defects. Consequently, positron annihilation and in particular the positron beam technique can provide information which is complementary to that provided by the techniques used for the analysis of composition and structure. A wealth of techniques is available for these latter purposes. Among them are Rutherford Backscattering (RBS), Nuclear Reaction Analysis (NRA), and Secondary Ion Mass Spectrometry (SIMS), which are capable of depth profiling of impurity atoms [1]. These techniques however lack sensitivity for detection of open volume defects. An indirect method for probing open volume are Small Angle Neutron or X-ray Scattering (SANS/SAXS). Open volume is best probed by allowing probe particles to be trapped in the cavities. Suitable particles are gas particles, helium and hydrogen, or positrons. Other techniques using a probe atom are Mössbauer and Perturbed Angular Correlation (PAC) [2], but these are better suited for probing very small cavities (1-5 vacancies). Using positrons as a probe has the advantage that the material is not damaged by the irradiation with the probe particles.

In layered structures obtained by various deposition techniques, open volume defects or microcavities are found with positrons $[3,4,5]$. The defects have their origin in the low mobility of the deposited atoms (low temperature deposition), imperfect regrowth during crystallization, stress relaxation in heterostructures, ion implantation damage, etc. Microcavities can be observed in measurements of the Doppler broadening of the $511 \mathrm{keV}$ annihilation line. With standard positron beams a zone $\sim 1 \mu \mathrm{m}$ deep below the material surface can be probed in order to reveal information 
on the depth distribution of the defects. Positron beam analysis with millimeter size beams has been developed since about 1982 (see the review by Schultz and Lynn [6]). In the future it is expected that micrometer size beams will be available so that lateral resolution can be achieved with the technique (see the contribution by Triftshäuser [7]). In this contribution we discuss the results we have obtained for silicon-based materials and for diamond films.

\section{EXPERIMENTAL}

The Delft Variable Energy Positron Beam (VEP) is employed for the analysis (figure 1). The magnetically guided beam delivers about $10^{5} \mathrm{e}^{+} \mathrm{s}^{-1}$ at the target. The beam diameter is about $8 \mathrm{~mm}$ [8,9]. Photon detection is performed by an intrinsic germanium detector with $1.1 \mathrm{keV}$ resolution at $511 \mathrm{keV}$ photon energy. The energy spectrum is recorded in 4096 channels. The Doppler broadening of the $511 \mathrm{keV}$ photopeak is characterized by the so-called $S$ (Shape) parameter [10] defined as the ratio between the area under the central part of the photopeak and the total area under the peak. Settings for our apparatus are such that $S$ for defect-free tungsten and silicon amounts to 0.45 and 0.63 respectively. In order to allow comparison with results obtained with other beams we frequently use the scaled $S$-parameter, $S_{\text {rel }}=S / S_{\text {defect free }}$. Also the positronium fraction and surface branching of positrons back-diffused to the surface are measured. A standard measurement consists of recording $S$ and the positronium fraction vs the positron energy $[6,8,9]$.

To transform the measured curves into practical information on depth profiles of defects and the nature of the defects, modelling and fitting is done with a computer program VEPFIT [11]. The program solves the rate and diffusion equations for positrons implanted according to a certain depth profile and determines the trapped or surface released fractions for a chosen model which may consist of a layered structure or a damage depth profile. Parameters that can be fitted are diffusion lengths, which are indicative of the defect concentrations in the layers, and S-parameters specified for the bulk, surface and defects in the layers.

Helium desorption spectra were recorded with a UHV-desorption spectrometer described in [12].

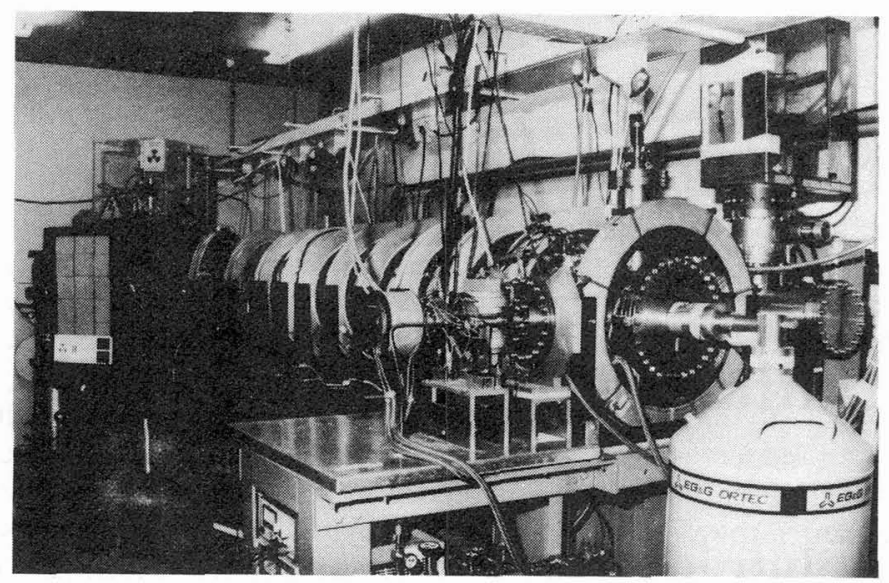

Fig. 1. Photograph of the VEP in its current configuration.

\section{EPITAXIAL SILICON}

Films grown by Molecular Beam Epitaxy (MBE) and Solid Phase Epitaxy (SPE) were examined by the positron beam technique [13]. The MBE sample was prepared by deposition at $1000 \mathrm{~K}$ during Si 
evaporation with a deposition rate of $0.3 \mathrm{~nm} \mathrm{~s}^{-1}$. The SPE layer was formed by deposition at room temperature (RT) of amorphous $\mathrm{Si}$, followed by an anneal treatment at $830 \mathrm{~K}$ causing solid phase epitaxial regrowth of the layer. The substrates had carbon contamination at the surface which eventually promoted the formation of defects. The results shown in figure 2 indicate considerable differences between the curves for the two epitaxial layers and the result expected for defect-free silicon. Analysis with VEPFIT shows that the layer contains open volume defects with a relative S-parameter of 1.06 at concentration levels of 500 and 30 appm for SPE and MBE respectively. The defects appeared to be below the detection limit of RBS. The results were compared with Transmission Electron Microscopy (TEM) results and with results obtained by probing the defects with variable energy helium implantation and thermal helium desorption spectrometry (THDS) ([14], and section 8). It appeared that two types of defects were present: voids with a diameter from 1 to $10 \mathrm{~nm}$ and much smaller defects at a much higher concentration, presumably associated with line or planar defects. The latter are present in both samples. Voids however are predominantly present in the SPE sample (concentration of the order of 2 appm).

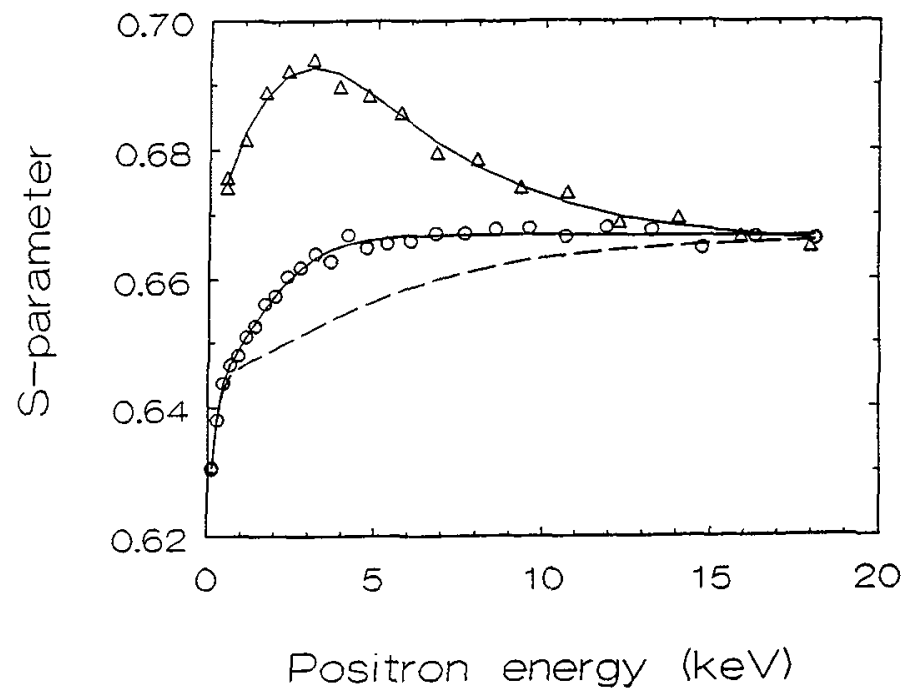

Fig. 2. The lineshape parameter $S$ vs the incident positron energy, measured at SPE ( $\Delta$ ) and MBE (o) grown epitaxial layers. The lines through the data were obtained using the program VEPFIT. The dashed curve represents defect-free Si.

\section{AMORPHOUS SILICON}

\subsection{Low-temperature vi. ur deposition}

In an MBE-system a-Si was grown as follows: $10 \mathrm{~nm}$ was deposited at RT on a silicon wafer, after which $300 \mathrm{~nm}$ was deposited at $250^{\circ} \mathrm{C}$. It was confirmed by Raman spectroscopy that a completely amorphous layer was grown. Positron annihilation results are shown in figure 3 . The curve can be fitted by a model consisting of a $400 \mathrm{~nm}$ thick top layer with voids $(S=1.141)$ and a diffusion length $\mathrm{L}$ of $5.4 \mathrm{~nm}$. Raman spectroscopy showed that the bond angle distortion was close to what is found for annealed and thus "defect-free" amorphous silicon. The $S$-parameter is very high, in fact among the highest measured for silicon. Thus, large voids must have been grown in otherwise "perfect" amorphous silicon. The high measured S-parameter is in accordance with the value of 1.15 that Simpson et al. [15] found for MBE silicon grown on $\mathrm{Si}(100)$ at a temperature just below $260^{\circ} \mathrm{C}$. 


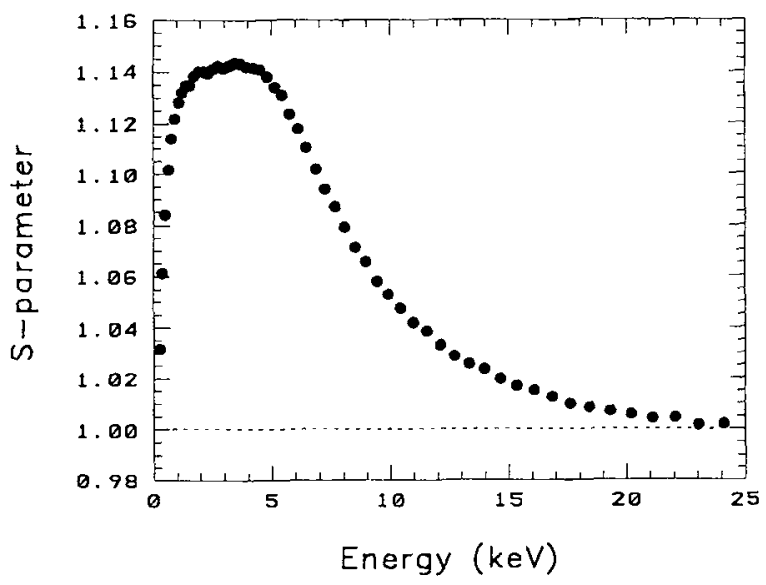

Fig. 3. Positron annihilation measurements on $M B E$-system grown amorphous silicon.

\subsection{PECVD hydrogenated silicon}

Hydrogenated amorphous silicon was deposited in a Plasma Enhanced Chemical Vapour Deposition (PECVD) process from a 1:1 volume ratio mixture of $\mathrm{SiH}_{4}$ and $\mathrm{H}_{2}$ at temperatures ranging from 5 to $200{ }^{\circ} \mathrm{C}$. Measurement results are shown in figure 4. It appears that the layer contains defects with $\mathrm{S}_{\mathrm{rel}}$ decreasing for higher deposition temperatures. It was also found that deposition with undiluted

Table 1. Fit results for the S-parameter and diffusion length (L) from the positron annihilation measurements on the a-Si:H samples, and the radius of gyration of the voids $\left(R_{g}\right)$ and void fraction $\left(v_{f}\right)$ derived from the SAXS data. $T_{s}$ is the substrate temperature during deposition

\begin{tabular}{|l|c|c|c|c|c|}
\hline \multicolumn{2}{|c|}{ growth conditions } & \multirow{2}{*}{$\begin{array}{c}S \\
\left( \pm 5 \times 10^{-4}\right)\end{array}$} & $\begin{array}{c}L(\mathrm{~nm}) \\
( \pm 0.6)\end{array}$ & $R_{g}(\mathrm{~nm})$ & $v_{f}$ \\
\hline \multicolumn{1}{|c|}{ gases } & $T_{s}\left({ }^{\circ} \mathrm{C}\right)$ & & & \\
\hline $\mathrm{SiH}_{4}$ & 50 & 1.0662 & 3.0 & 0.65 & 0.2521 \\
$\mathrm{SiH}_{4}$ & 100 & $\ldots$ & $\ldots$ & 0.59 & 0.1002 \\
$\mathrm{SiH}_{4}$ & 200 & 1.0327 & 5.0 & 0.54 & 0.0239 \\
$\mathrm{SiH}_{4} / \mathrm{H}_{2}$ & 50 & 1.0651 & 2.4 & 0.59 & 0.1168 \\
$\mathrm{SiH}_{4} / \mathrm{H}_{2}$ & 83 & $\ldots$ & $\ldots$ & 0.57 & 0.0679 \\
$\mathrm{SiH}_{4} / \mathrm{H}_{2}$ & 100 & 1.0534 & 2.4 & $\ldots$ & $\ldots$ \\
$\mathrm{SiH}_{4} / \mathrm{H}_{2}$ & 125 & $\ldots$ & $\ldots$ & 0.57 & 0.0447 \\
$\mathrm{SiH}_{4} / \mathrm{H}_{2}$ & 150 & 1.0364 & 3.3 & 0.52 & 0.0274 \\
$\mathrm{SiH}_{4} / \mathrm{H}_{2}$ & 200 & 1.0273 & 6.2 & 0.48 & 0.0167 \\
\hline
\end{tabular}

$\mathrm{SiH}_{4}$ led to slightly higher $\mathrm{S}$-values. In table 1 results are given of the $\mathrm{S}$-parameters and diffusion lengths $\mathrm{L}$ obtained from fitting. SAXS measurements are also included. They reveal a decrease of cavity size from $1.2 \mathrm{~nm}$ at $50{ }^{\circ} \mathrm{C}$ to $0.96 \mathrm{~nm}$ at $200{ }^{\circ} \mathrm{C}$ deposition temperature. Void fractions were reduced in this temperature interval from $11 \%$ to $1.7 \%$. This is in line with the observed increase of the diffusion length. The value of 1.06 for the S-parameter is much lower than found for the cavities in the low temperature MBE samples. In section 9 a possible explanation will be given for this observation. 


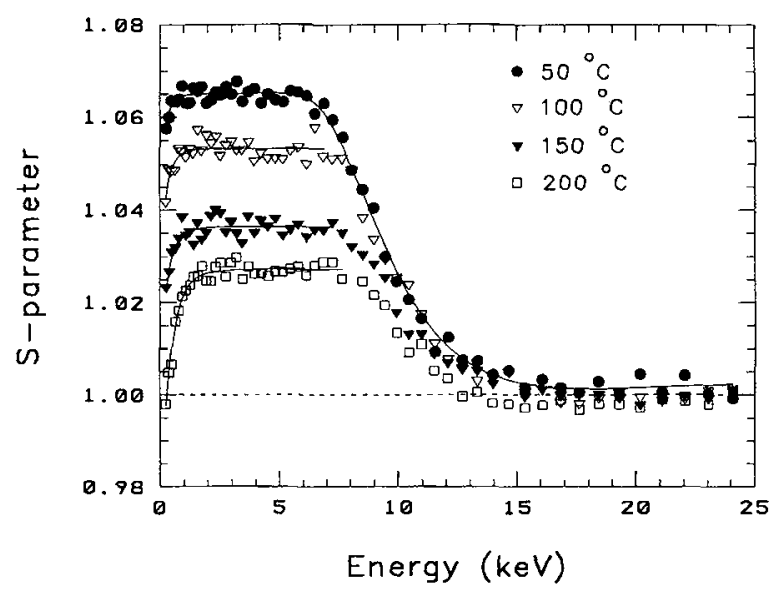

Fig. 4. Positron-annihilation measurements on PECVD-grown hydrogenated amorphous silicon; deposition from a 1:1 mixture of $\mathrm{SiH}_{4} / \mathrm{H}_{2}$ at 50, 100, 150 and $200{ }^{\circ} \mathrm{C}$.

\subsection{Sputter-deposited silicon}

Krypton-sputtered amorphous silicon films were prepared by sputtering on a Si substrate in a low pressure (0.01-0.1 Pa) krypton plasma [16]. The growing silicon layer which was kept at $310{ }^{\circ} \mathrm{C}$ was irradiated with a flux of low energy $(50 \mathrm{eV})$ krypton ions which could be varied so that $\mathrm{Si}$ atom/ $\mathrm{Kr}$ atom arrival ratios from 0.01 to 3.0 could be achieved at the growing film. The krypton bombardment resulted in incorporated $\mathrm{Kr}$ concentrations from 0.5 to 5 atomic \%. Due to the extra kinetic energy of the sputtered $\mathrm{Si}$ atoms and the extra energy deposited by the impinging $\mathrm{Kr}$ ions, the growing layer did not contain large cavities of the kind observed in the above examples. $S$ parameters shown in figure 5 decreased with increased $\mathrm{Kr}$ concentration from 1.024 to 1.018 which is lower than

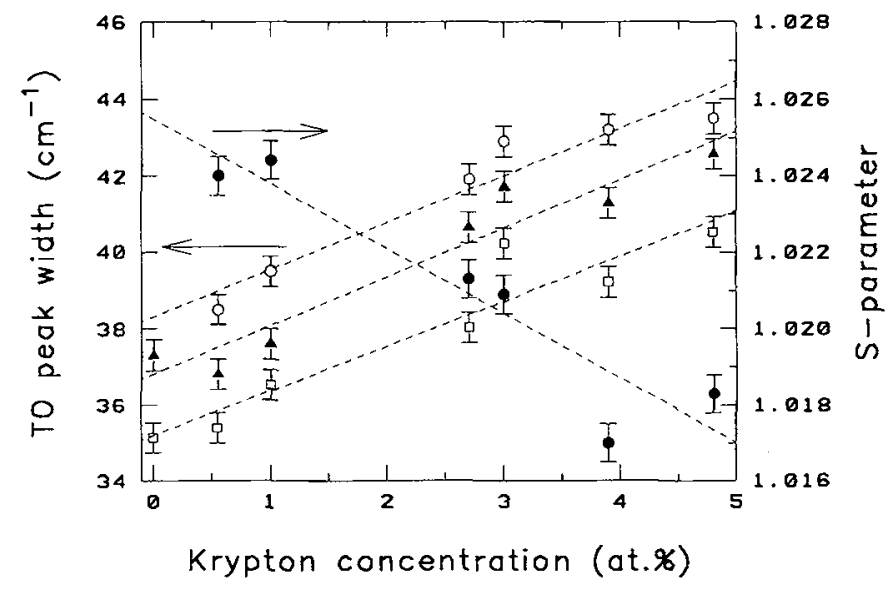

Fig. 5. S-parameter (•) and TO-peak width (०) of the a-Si:Kr films as a function of the krypton concentration. The widths of the TO-peak after relaxation at $310^{\circ} \mathrm{C}(\Delta)$ and $480{ }^{\circ} \mathrm{C}(\square)$ are also plotted. The data for $0 \%$ krypton indicate the TO-peak widths of ion-beam amorphized silicon relaxed at $310^{\circ} \mathrm{C}$ and $480^{\circ} \mathrm{C}$ respectively. The dashed lines are to guide the eye. 
found for an amorphous layer amorphized by $1 \mathrm{MeV}$ Si ion bombardment at $20 \mathrm{~K}$. The bond angle distortion measured by Raman spectroscopy was considerably larger for the samples with high $\mathrm{Kr}$ concentration (figure 5). Also, compressive stresses in the films with high $\mathrm{Kr}$ concentration were higher. Annealing to $480{ }^{\circ} \mathrm{C}$ led to a reduction of bond angle distortion. The results all point to a material with only very small cavities corresponding to those found in "perfect" amorphous silicon with $\mathrm{Kr}$ atoms incorporated in small clusters causing the observed extra stress and bond angle distortion [17].

\section{GAS INTERACTION WITH CAVITIES}

From earlier combined THDS and TEM observations of $\mathrm{keV}$ heliumion irradiated silicon $[18,19]$ it was found that voids in silicon can be created by annealing the sample to a temperature of approximately 800 ${ }^{\circ} \mathrm{C}$. In the temperature range from $100{ }^{\circ} \mathrm{C}$ to $700{ }^{\circ} \mathrm{C}$ helium bubbles nucleate and grow; at $800{ }^{\circ} \mathrm{C}$ the bubbles formed lose all the helium by permeation of the helium through the silicon layer separating the bubbles from the sample surface. For $2.5 \mathrm{keV} \mathrm{He}$ ions at a dose of $1.6 \times 10^{16} \mathrm{He} \mathrm{cm}{ }^{-2}$, the $\mathrm{He}$ concentration is sufficiently high that microcavities larger than $1 \mathrm{~nm}$ form during thermal annealing. Examining the sample with positron beam analysis gives the results shown in figure 6a. The S-value has increased dramatically. A value of 1.12 is obtained by fitting of the data. A sample with these cavities is of interest for studying the interaction with helium and hydrogen gas. For this purpose the samples were again irradiated with helium and hydrogen. In figure $6 \mathrm{~b}$ the result of hydrogen decoration and of the subsequent annealing are shown. A dramatic reduction of the S-parameter to 1.03 is observed for hydrogen. The S-parameter is reduced to 1.05 upon decoration with $\mathrm{He}$. In both cases the high S-value is fully recovered after annealing to $800{ }^{\circ} \mathrm{C}$. This is in agreement with the expected fast permeation of helium and hydrogen from the cavities at this temperature. The experiments demonstrate that the state which the positrons occupy in the cavities is

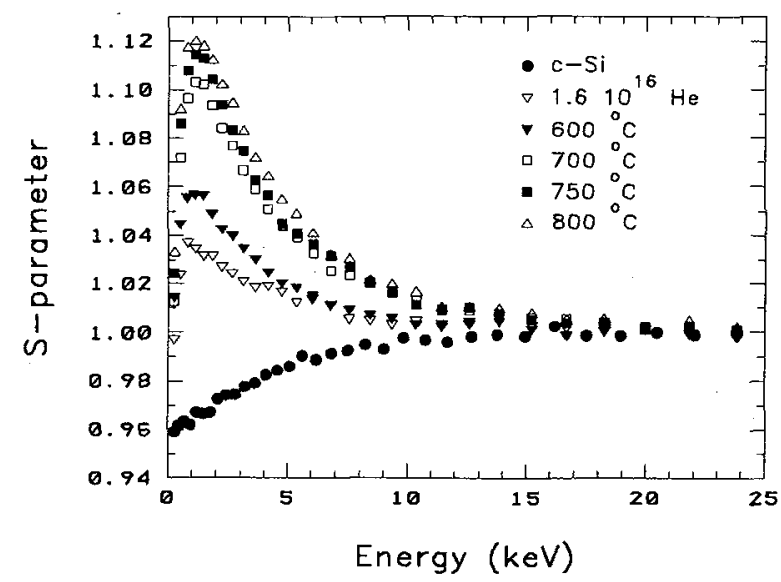

Fig. 6a. Positron annihilation measurements of a $2.5 \mathrm{keV}$ helium implantation into c-Si to a dose of $1.6 \times 10^{16} \mathrm{~cm}^{-2}$ and after subsequent anneals at 600,700,750, and $800^{\circ} \mathrm{C}$.

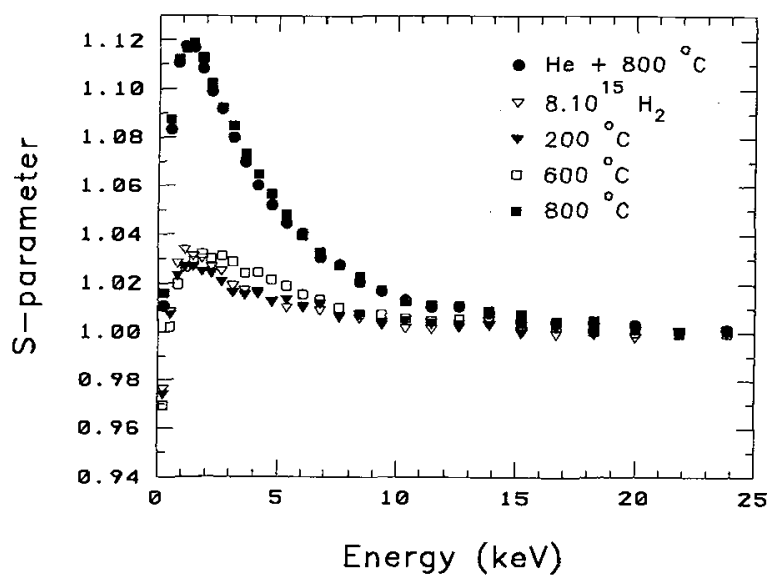

Fig. 6b. Decoration of voids in c-Si by a $2.5 \mathrm{keV}$ hydrogen implantation to a dose of $8 \times 10^{15} \mathrm{~cm}^{-2}$ and after anneals at 200,600 , and $800{ }^{\circ} \mathrm{C}$. 
nearly fully suppressed by the presence of gas. In the case of helium there is no surface binding; thus the increase of the gas density in the cavity volume plays a role. For hydrogen the Si-H bonds at the inner surface of the cavity may have an additional effect.
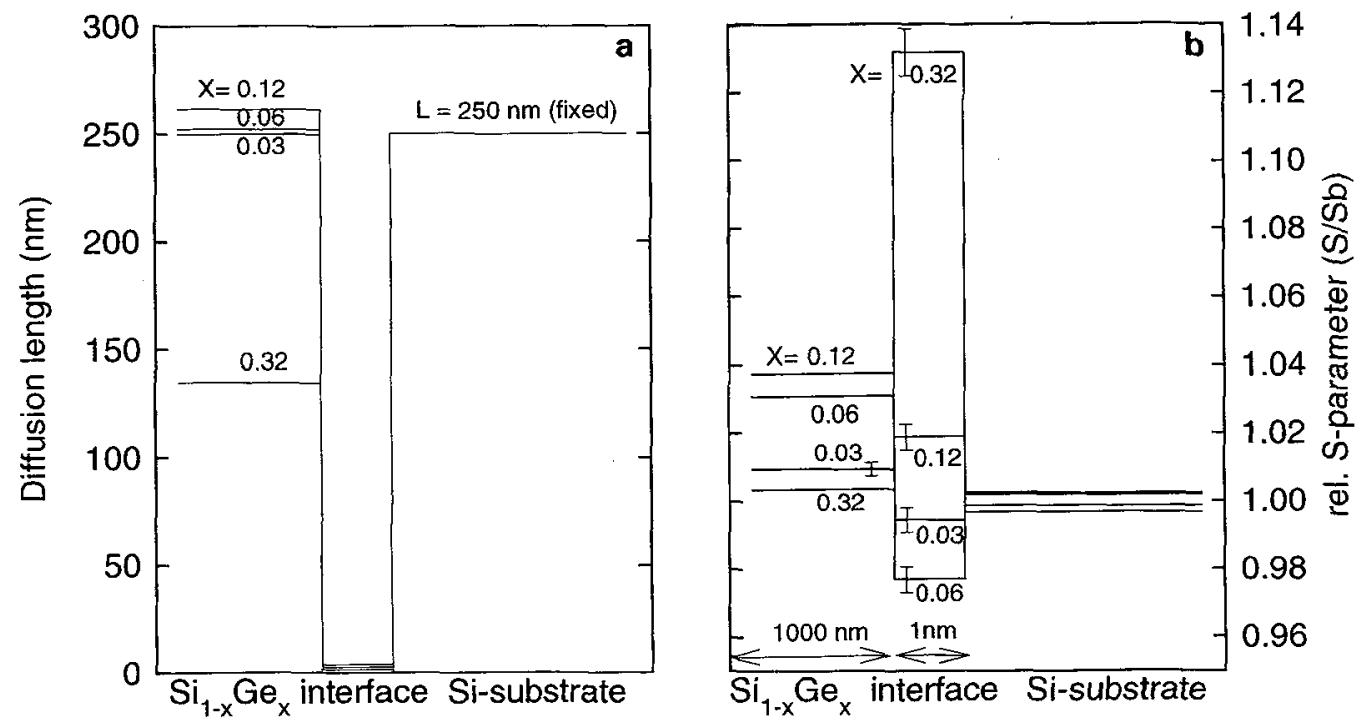

Fig. 7. Results of model fitting with VEPFIT for a Ge-Si/Si layered structure. a) Diffusion length vs depth, b) relative $S$-parameter vs depth.

\section{LAYERED STRUCTURES AND INTERFACES}

Examples of defects in epitaxial layers of $\mathrm{CoSi}_{2}$ on silicon are presented and discussed by Reader et al. [20] and $\mathrm{La}$ Via et al. [21]. Here we present results on layered GeSi heterostructures. Layers with composition $\mathrm{Ge}_{\mathrm{x}} \mathrm{Si}_{1-\mathrm{x}}$ can be grown on a Si-substrate without strain relaxation for a limited germanium concentration and thickness of the film [22]. The lattice mismatch is adapted by an increase of the lattice parameter in the growth direction of the film. Positron measurements and subsequent analysis on a series of $100 \mathrm{~nm}$ thick films with $\mathrm{x}$ varying from 0.02 to 0.32 yielded the diffusion lengths and $S$-parameter values shown in figs. $7 \mathrm{a}$ and $\mathrm{b}$. The limit for strain relaxation of a film with this thickness deposited at a temperature of $600{ }^{\circ} \mathrm{C}$ is slightly beyond $\mathrm{x}=0.12$. The positron results show clearly that the film with $x=0.32$ has undergone relaxation. The positron diffusion length has been reduced to half the value found for the films containing less Ge, which indicates the presence of trapping centers presumably caused by the matching dislocations which are formed. There is no large effect on the S-parameter of the film. Apparently the defects do not have the character of large open volumes. It should be noted that the composition change with respect to $S$ might have led to an extra change of $S$ in addition to the effect due to the defects. A large increase of the $S$-parameter at the interface is however observed. The high value of $S$ indicates open volume defects right at the interface. As yet, no microscopic observations are available to confirm that cavities are present at the interface but one might speculate that the high dislocation density at the surface has caused intersections of dislocations (dislocation network) with a strongly enhanced volume for positron trapping.

\section{DIAMOND LAYERS}

Diamond deposition by plasma enhanced CVD techniques is a rapidly growing activity with applications in the field of wear resistant coatings and on semiconductor materials. Positron analysis of diamond layers has revealed the presence of cavities in these materials. It appears that the cavity formation strongly depends on the fraction of $\mathrm{CH}_{4}$ in the deposition gas. Cavities in PECVD depo- 
sited diamond films are easily formed when the composition of the deposition gas is not ideal. In figure 8 positron depth profiling results are shown for a series of $700 \mathrm{~nm}$ thick diamond films on silicon produced for concentrations of $\mathrm{CH}_{4}$ in the $\mathrm{H}_{2}$ gas varying from $0.25 \%$ to $2 \%$. Deposition temperatures were about 850 ${ }^{\circ} \mathrm{C}$. It appears that a high methane concentration leads to cavities with $\mathrm{S}_{\mathrm{rel}}=$ 1.16 (with respect to silicon) and $S_{\text {rel }}=$ 1.41 with respect to defect-free diamond. Similar results were found by Uedono et al. [23]. This high value is comparable with cavities found in PECVD deposited hydrogenated silicon or in low temperature MBE silicon. Apparently, in the case of the high deposition rate caused by the high methane concentration, cavities can develop more easily. Measurements on graphite and diamondlike carbon reveal $S$ values lower than observed for the diamond films. The films are real diamond, as was derived from positron re-emission measurements. All the films re-emitted low energy implanted positrons, whereas graphite and diamondlike carbon did not.

The results show that positron beam analysis could turn into a very useful contactless and non-destructive tool for quality control of growing diamond films.

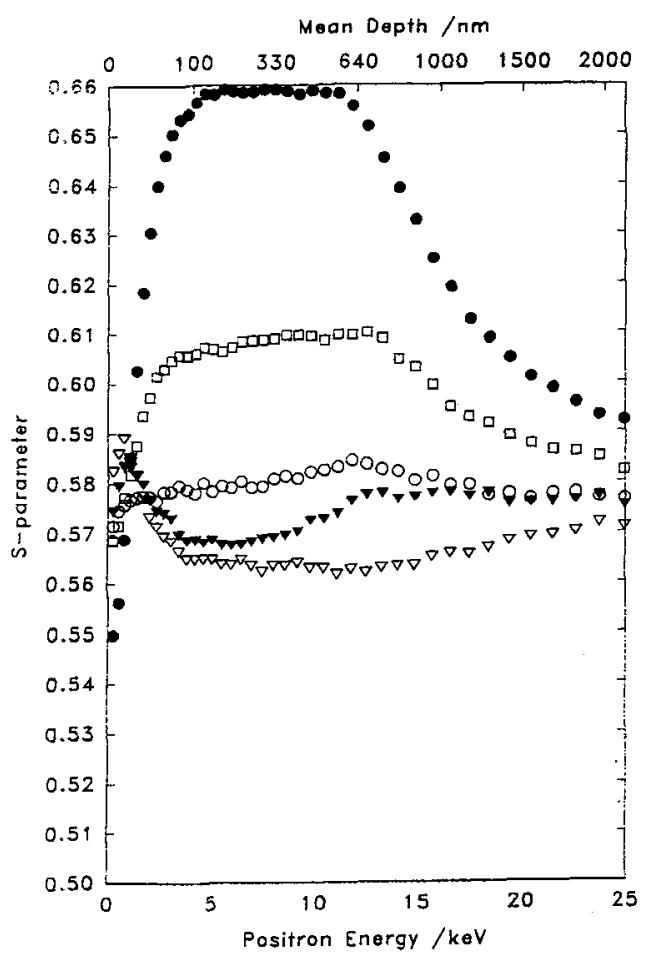

Fig. 8. The methane concentration in the deposition gas and the substrate temperature were varied from $2.0 \% / 850{ }^{\circ} \mathrm{C}(\bullet), 1.0 \% / 850{ }^{\circ} \mathrm{C}$ (口), $0.5 \% / 850{ }^{\circ} \mathrm{C}(0)$, $0.5 \% / 750{ }^{\circ} \mathrm{C}(\nabla)$ to $0.25 \% / 900{ }^{\circ} \mathrm{C}(\nabla)$, respectively.

\section{HELIUM AND HYDROGEN PROBING OF DEFECTS}

Similarly to positrons, helium can be implanted into a material. By virtue of the high room temperature mobility and low solubility of helium in a large number of materials, e.g., in most metals, the helium will be easily trapped in cavities and will dissociate only when the temperature is increased. The technique for helium decoration and desorption is called thermal helium desorption spectrometry (THDS). Most of the results have been obtained for defects in metals but here we discuss results on epitaxially grown silicon layers (see also section 3). In figure 9 a series of helium desorption spectra is shown obtained by varying the implantation energy of the helium. Several peaks contribute to the spectra, e.g., one at $400 \mathrm{~K}$ due to helium release by interstitial diffusion, peaks at $600-850 \mathrm{~K}$ due to small vacancy clusters, and a peak at $1100 \mathrm{~K}$ due to cavities $>1 \mathrm{~nm}$ diameter. From the range profile of the implanted helium and the peak populations defect concentrations were derived [14].

\section{DISCUSSION}

\subsection{Defects on a relative S-parameter scale}

From a large number of studies on defects in silicon by different groups it has been derived which specific $S$-parameters must be assigned to certain defects. In terms of the relative $S$-parameter it has been found that $S_{\text {rel }}$ takes on the following values [24]: for monovacancies 1.030, divacancies 1.0341.038 , vacancy clusters $1.047-1.061$, and voids $1.10-1.14$. Thus, $S_{\text {rel }}$ increases with the size of the 
cavity. When oxygen is associated with the vacancy clusters the S-parameter becomes smaller than for bulk silicon: 0.900.978 . For amorphous silicon $S_{\text {rel }}=1.02-1.04$; therefore, it corresponds to positron trapping in small cavities. Methods which are able to measure the Doppler broadening effect with a higher resolution, such as $2 \mathrm{D} \mathrm{ACAR}$, although more time consuming, allow the characterization of the defects in more detail [25].

\subsection{A simple model for positronium in empty and gas-filled cavities}

Positron annihilation behaviour in cavities can partly be ascribed to the formation of positronium $\left(\mathrm{P}_{s}\right)$, a bound $\mathrm{e}^{+}-\mathrm{e}^{-}$ pair. A simple model for positronium in a microcavity of radius $r$ can be obtained under the assumption that the interaction of the positronium atom with its surroundings can be described as a quantum mechanical particle in a three-dimensional rectangular potential well [26]. Since positronium cannot exist in the bulk of metals and silicon (the binding energy equals zero), the potential well has a depth $V_{o}$ which is larger than the positronium work function, i.e. for silicon:

$$
V_{o}>\left|\phi_{P s}\right| \approx 2 \mathrm{eV}
$$

(see Schultz and Lynn, [6]). Thus, as has been indicated in the insert of figure 10, positronium will dissociate and be absorbed as a separate bulk positron and valence electron when the energy of the positronium atom exceeds $\phi_{\mathrm{P}_{\mathrm{s}}}$.

An estimate of the interaction radius $r_{P s}^{\prime}$ of positronium with the wall of the cavity is $0.1 \mathrm{~nm}$, i.e., twice the radius of the positronium atom. The energy levels have been calculated for $V_{o}=5 \mathrm{eV}$ but the energies appear to be rather insensitive to changes in the depth of the potential well (Stewart et al. [26]).

It appears from figure 10 that positronium in the ground state has no binding to cavities with a radius smaller than $0.35 \mathrm{~nm}$ (the binding energy becomes negative), which corresponds to voids consisting of at most nine vacancies in silicon. Taking into account thermally activated dissociation of positronium during its lifetime, the radii of the cavities in which positronium can survive are even somewhat larger.

When placed in gaseous surroundings which exert a hydrostatic pressure $P$ on the particle, positronium will counterbalance this pressure by adapting its volume $V_{P s}$ to a value given by:

$$
P=-\frac{d E_{n}}{d V_{P S}}=-\frac{1}{4 \pi r^{2}} \frac{d E_{n}}{d r}
$$

The curve for positronium in the ground state $(n=0)$ is shown in figure 10 from which it can be derived that positronium will not exist in a gas bubble at pressures beyond $\sim 1 \mathrm{GPa}$. Taking into account thermally activated detrapping, stable energy levels will be $0.3 \mathrm{eV}$ lower than $\phi_{\mathrm{Ps}}$ and thus the pressure will also be lower, i.e. $\sim 0.7 \mathrm{GPa}$.

This simple calculation explains rather well the results obtained for the gas decoration of the 


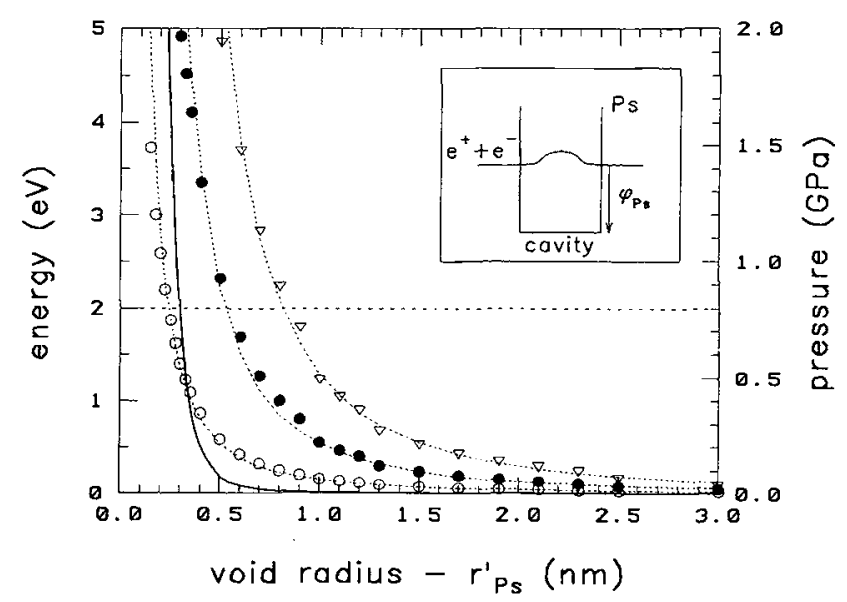

Fig. 10. Energy levels of positronium with respect to the bottom of a three-dimensional rectangular potential well (see the insert) as a function of the cavity radius minus the radius of the positronium atom. The ground state level $(n=0: 0)$ and higher excitation levels are plotted. The solid line represents the pressure exerted by the positronium atom on its surroundings.

cavities in the implantation experiments of section 5 . The cavities releasing helium at $800{ }^{\circ} \mathrm{C}$ have a radius which is clearly larger than $1 \mathrm{~nm}$ [27]. When the cavities are devoid of helium or hydrogen gas, bound positronium may easily exist in the open volume. However, when the same amount of gas as used for the creation of the voids is re-implanted, the cavities will be pressurized again to a level equal to or higher than the level during creation. The reason is that no annealing takes place during the re-implantation experiment and therefore all the helium is available for decoration of the cavities.

The Doppler broadening is considerably reduced (i.e., the S-parameter is increased) with respect to pure silicon when the cavity diameter becomes larger than a critical diameter of $0.9 \mathrm{~nm}$. The effect may be attributed to the creation of positronium. It was demonstrated that cavities filled with gas (He, $\mathrm{Ar}$, hydrogen) exhibit an increase of the Doppler broadening because positronium formation is suppressed by the gas pressure.

\section{CONCLUSIONS AND FINAL REMARKS}

Examples have been given of the detection of microcavities in materials (semiconductor materials) by means of particle probe techniques. It has been shown that, in particular, positrons can be employed for non-destructive testing of thin films and interfaces. The helium desorption technique is even more sensitive for defect characterization and can identify more defect types. However, the method is nondestructive only when the defects are stable during thermal desorption.

Positron techniques have a wider application with regard to material choice and can be used as a micro-analysis technique. When in the future positron microbeams become available, the lateral resolution can be pushed into the micrometer range.

\section{Acknowledgement}

We acknowledge the technical assistance by J. de Roode, K.T. Westerduin and K. Roos.

\section{REFERENCES}

[1] Feldmann, L.C., and J.W. Mayer, Fundamentals of Surface and Thin Film Analysis (Elsevier Science Publishing Co., Inc., Amsterdam, 1986). 
[2] Waard, H. de, F. Pleiter, L. Niesen, and D.W. Hafemeister, Hyperfine Interactions, 10 (1981) 643.

[3] Jackman, T.E., G.C. Aers, M.W. Denhoff, and P.J. Schultz, Appl. Phys. A, 49 (1989) 335.

[4] Asoka-Kumar, P., H.-J. Gossmann, F.C. Unterwald, L.C. Feldman, T.C. Leung, H.L. Au, V. Talyanski, B. Nielsen, and K.G. Lynn, Phys. Rev. B48 (1993) 5345.

[5] Schut, H., A. van Veen, G.F.A. van der Walle, and A.A. van Gorkum, Appl. Phys. 70 (1991) 3003.

[6] Schultz, P.J., and K.G. Lynn, Rev. Mod. Phys., 60 (1988) 701.

[7] W.Triftshäuser, in this volume.

[8] Schut, H., Thesis, Delft University of Technology, Delft (1990).

9] Veen, A. van, J. Trace and Microprobe Techniques, 8 (1990) 1.

[10] MacKenzie, I.K., J.A. Eady, and R.R. Gingerich, Phys. Lett. A, 33(1970) 279.

[11] Veen, A. van, H. Schut, J. de Vries, R.A. Hakvoort, and M.R. IJpma, in: Positron Beams for Solids and Surfaces, AIP Conf. Proc., 218, eds. P.J. Schultz, G.R. Massoumi, and P.J. Simpson (AIP, New York, 1990) p. 171.

[12] Veen, A. van, A. Warnaar, and L.M. Caspers, Vacuum, 30 (1980) 109.

[13] Schut, H., A. van Veen, G.F.A. van de Walle, and A.A. van Gorkum, J. Appl. Phys., 70 (1991) 3003.

[14] Veen, A. van, A.H. Reader, D.J. Gravesteijn and A.A. van Gorkum, Thin Solid Films, 24 (1993) 206.

[15] Simpson, P.J., P.J. Schultz, T.E. Jackman, G.C. Aers, J.P. Noel, D.C. Houghton, D.D. Perovic, and G.C. Weatherly, in: Positron Beams for Solids and Surfaces, AIP Conf. Proc., 218, eds. P.J. Schultz, G.R. Massoumi, and P.J. Simpson (AIP, New York, 1990) p. 125.

[16] Greuter, M.J.W., L. Niesen, R.A. Hakvoort, J. de Roode, A. van Veen, A.J.M. Berntsen, and W.G. Sloof, Hyperfine Interactions 79 (1993) 669.

[17] Greuter, M.J.W., Thesis, Groningen University, Groningen (1993).

[18] Evans, J.H., A. van Veen, and C.C. Griffioen, Nucl. Instr. and Meth. B 28 (1987) 360.

[19] Griffioen, G.C., J.H. Evans, P.C. de Jong, and A. van Veen, Nucl.Instr. and Meth. B 27 (1987) 417.

[20] Reader, A.H., H. Schut, R.A. Hakvoort, and A. van Veen, in this volume.

[21] La Via, F., C. Spinella, A.H. Reader, J.P.W.B. Duchateau, R.A. Hakvoort, and A. van Veen, Appl. Surface Sci. 73 (1993) 108.

[22] Bean, J.C., Mat. Res. Soc. Symp. Proc. 37 (1985) 245.

[23] Uedono, A., S. Tanigawa, H. Funamoto, A. Nishikawa and K. Takahashi, J. Appl. Phys. 29 (1990) 555 .

[24] Hakvoort, R.A., Thesis, Delft University of Technology, Delft (1993).

[25] Rozing, G.J., A.W. Weeber, P.E. Mijnarends, A. van Veen, J. de Vries, H.Schut, Mater. Sci.Forum 105-110 (1992) 1205.

[26] Stewart, A.T., C.V. Briscoe, and J.J. Steinbacher, Can. J. Phys., 68 (1990) 1362.

[27] Veen, A. van, in: Fundamental Aspects of Inert Gases in Solids, NATO ASI Series B, 279, eds. S.E. Donnelly, and J.H. Evans (Plenum Press, New York, 1991) p. 41. 